\section{Kennen Sie die Lobomykose?}

Ein 37-jähriger Mann lebte seit zwölf Jahren mit merkwürdigen Hautveränderungen am rechten Ohr. Im Bereich der Helix und der Anthelix zeigten sich mehrere hautfarbene Knoten mit glatter Oberfläche und bindegewebiger Konsistenz.

$\mathrm{D}$ ie Veränderung erinnerte auf den ersten Blick an ein Narbenkeloid (Abb. 1 a und b). Der Mann erinnerte sich allerdings nicht an ein Trauma der Ohrmuschel. Er hatte viele Jahre als Kautschuksammler in Tefé gelebt, einer Stadt in der Amazonasregion Brasiliens.

Eine Biopsie aus einem der Knoten ließ multiple Granulome erkennen, die zahlreiche rundliche pilzartige Strukturen enthielten. Der Befund war vereinbar mit Lacazia loboi (Abb. 1 c, Grocott-Gomori Methaminsilber-Färbung). Die Gewebekultur ließ allerdings kein Pilzwachstum
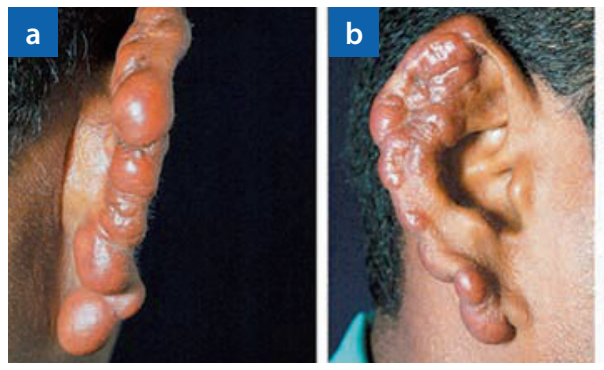

erkennen. Die Ärzte stellten aufgrund des klinischen und histologischen Befundes die Diagnose einer Lobomykose. Die Knoten sollten operativ entfernt werden, doch verweigerte der Patient eine Operation. Eine Therapie mit Ketoconazol hatte keinen wesentlichen Effekt.

Fazit: Die Lobomykose gehört zu den häufig vorkommenden tiefen Mykosen in den tropischen Regenwaldgebieten Südamerikas und wurde erstmals 1931von dem brasilianischen Dermatologen Jorge Lobo beschrieben. Es handelt sich um

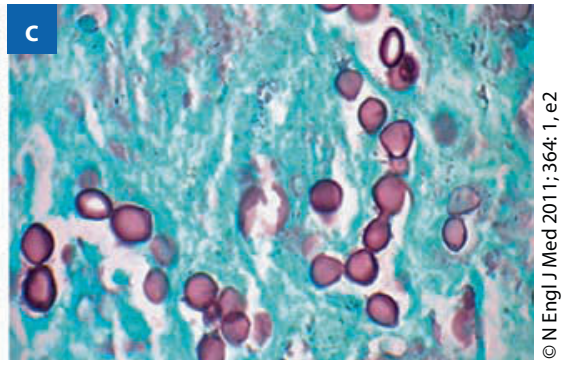

eine subkutane Pilzinfektion nach minimalen Traumata der Haut. Betroffen sind überwiegend Fischer und Landarbeiter in den Flusslandschaften Amazoniens. Die chronische und meist über Jahre verlaufende Erkrankung manifestiert sich am häufigsten im Bereich der Extremitäten, aber auch im Gesicht und hier bevorzugt an den Ohrmuscheln.

Sie beginnt üblicherweise mit einem zunächst schmerzhaften glänzenden Knoten. Im weiteren Verlauf breiten sich die Knoten aus und konfluieren, wodurch ein Hautbefund entsteht, der an ein Keloid erinnert. Diese Knoten werden meistens frühzeitig chirurgisch entfernt, um die kosmetische Entstellung durch den weiteren Verlauf zu verhindern. Der Erreger der Lobomykose, Lacazia loboi (syn. Loboa loboi), ist diagnostisch im Nativpräparat und in der Histologie einfach nachzuweisen, die kulturelle Anzüchtung ist aber bis heute nicht gelungen

Prof. Dr. Hermann FüeßI

Francesconi F und V. Lobomycosis.

New Engl J Med 2011; 364: 1, e2

Abb. 1: Keloidartige Veränderungen bei Lobomykose $(\mathrm{a}, \mathrm{b})$, histologisches Bild (c)

\section{Vitiligo: Zelltransplantation ist auch bei Kindern erfolgreich}

Die Verpflanzung eigener Zellen auf Hautareale mit Vitiligo hat sich bei Erwachsenen bereits bewährt. Indische Dermatologen haben diese Methode nun auch bei Kindern getestet.

\footnotetext{
A der Untersuchung nahmen 13 Kinder und Jugendliche im Alter von 8-17 Jahren teil. Die Erkrankung war seit mindestens einem Jahr stabil. Behandelt wurden insgesamt 19 Hautstellen.

Die Ärzte entnahmen Spalthaut seitlich am Oberschenkel und fertigten daraus eine Suspension von Melanozyten und Keratinozyten der basalen Epidermis an. Die Empfängerstelle - zwischen drei- und zehnmal größer als das entnommene Hautstück - bereiteten sie mit einer Dermabrasion vor. Alle Maßnahmen erfolgten unter Lokalanästhesie. Nach acht
}

Tagen wurden die Verbände abgenommen. Die Patienten waren angewiesen, die Stellen mit den Zelltransplantaten täglich der Mittagssonne für maximal 30 Minuten auszusetzen.

15 der 19 Läsionen wiesen nach einem Jahr Repigmentierungsraten von über $90 \%$ auf, die übrigen vier Hautareale zeigten Raten zwischen 75-90\%. Alter, Geschlecht, Körperregion und Dauer der Erkrankung hatten in dieser Studie keinen Einfluss auf die Ergebnisse. Wesentliche Nebeneffekte der Therapie seien nicht zu beobachten gewesen, betonen die Auto- ren. Nur an einer der behandelten Läsionen entwickelte sich eine Infektion möglicherweise in Zusammenhang mit mangelnder Compliance.

Fazit: Die Verpflanzung eigener Hautzellen in Form einer epidermalen Suspension hat sich in dieser Studie auch bei Kindern und Jugendlichen als erfogreiche Therapie einer stabilen Vitiligo bewährt. Einschränkend zu erwähnen ist die geringe Fallzahl. Dennoch könnte diese Form der chirurgischen Behandlung zumindest für solche Patienten eine Option darstellen, bei denen andere Behandlungen nicht anschlagen

Dr. Robert Bublak

Sahni K et al. Noncultured epidermal suspension transplantation for the treatment of stable vitiligo in children and adolescents. Clin Exp Dermatol 2011; 36: 607-12 